\title{
Weight status and the perception of body image in men
}

This article was published in the following Dove Press journal:

Psychology Research and Behavior Management

25 July 2014

Number of times this article has been viewed

\section{Rick M Gardner \\ Department of Psychology, University of Colorado Denver, Denver, CO, USA}

Correspondence: Rick M Gardner

Department of Psychology,

University of Colorado Denver,

Campus Box 173, PO Box 173364,

Denver, CO 80217-3364, USA

$\mathrm{Tel}+\mathrm{I} 303556835$ I

Fax +l 3035563520

Email rick.gardner@ucdenver.edu
Abstract: Understanding the role of body size in relation to the accuracy of body image perception in men is an important topic because of the implications for avoiding and treating obesity, and it may serve as a potential diagnostic criterion for eating disorders. The early research on this topic produced mixed findings. About one-half of the early studies showed that obese men overestimated their body size, with the remaining half providing accurate estimates. Later, improvements in research technology and methodology provided a clearer indication of the role of weight status in body image perception. Research in our laboratory has also produced diverse findings, including that obese subjects sometimes overestimate their body size. However, when examining our findings across several studies, obese subjects had about the same level of accuracy in estimating their body size as normal-weight subjects. Studies in our laboratory also permitted the separation of sensory and nonsensory factors in body image perception. In all but one instance, no differences were found overall between the ability of obese and normal-weight subjects to detect overall changes in body size. Importantly, however, obese subjects are better at detecting changes in their body size when the image is distorted to be too thin as compared to too wide. Both obese and normal-weight men require about a 3\%-7\% change in the width of their body size in order to detect the change reliably. Correlations between a range of body mass index values and body size estimation accuracy indicated no relationship between these variables. Numerous studies in other laboratories asked men to place their body size into discrete categorizes, ranging from thin to obese. Researchers found that overweight and obese men underestimate their weight status, and that men are less accurate in their categorizations than are women. Cultural influences have been found to be important, with body size underestimations occurring in cultures where a larger body is found to be desirable. Methodological issues are reviewed with recommendations for future studies.

Keywords: body size, men, weight status, body image, body perception, literature review

\section{Introduction}

In this article, the role that weight status plays in the self-perception of men's bodies is reviewed. This is of importance because the inability to identify the distortion of one's perceived body image may become a predictive factor of subsequent overweight or obesity. ${ }^{1}$ The ability to identify such perceptual failures may also contribute to an ability to make an early diagnosis of eating disorders in young, healthy men. ${ }^{2}$ Furthermore, one's awareness of being overweight or obese has been shown to be an essential factor for successfully losing weight in both men and women. ${ }^{3}$

It is important to define precisely what is meant by "perception" in this review. Historically, the traditional view of human visual perception is that it is the process of viewing basic visual properties or sensory inputs without any direct influence 
from higher-level cognitive states - for example, attitudes, motives, expectations, and so on. ${ }^{4}$ Contemporary body image researchers have adopted an alternative view of perception, which was described by Thompson et $\mathrm{al}^{5}$ as the integrative model. Perception is viewed as that which is seen or recognized on a conscious level. It includes sensations that are detected by the sense organs, as well as via cognition. These cognitions may reflect an individual's beliefs, motivations, prior knowledge, and attitudes. ${ }^{5}$ Thus, both sensations and perceptions can affect how an individual sees his or her body on a conscious level. A few studies have examined the role of the sensory and nonsensory components separately. ${ }^{4}$ These studies will be described later.

The two most frequently investigated aspects of body image include how accurately body size is perceived, and feelings of dissatisfaction about one's body size and shape. ${ }^{4}$ In the body image literature, these are defined as the perceptual and affective components. A majority of the research in body image has focused on young females. ${ }^{4,5}$ This is undoubtedly due to the findings that both distorted body size perceptions, as well as dissatisfaction with body size and shape are related to eating disorders, which are more predominant in young females. ${ }^{5,6}$ Distortions in size perception, as well as body dissatisfaction, are commonly referred to as body image disturbance. The increasing incidence of eating disorders in young men in recent years has resulted in an increasing number of studies that have focused on this topic. ${ }^{7}$ In a recent review of the literature on eating disorders in men, ${ }^{7}$ it was found that $10 \%-20 \%$ of the cases of anorexia nervosa and bulimia nervosa, and up to $40 \%$ of cases of binge eating disorders, occur in men. ${ }^{7}$ Inaccurate perceptions of body size have been consistently found in women with anorexia, ${ }^{5}$ although the role in men has not been fully investigated. Also, the role that weight status has on eating disorders in men requires further investigation.

This review will emphasize the role that weight status has on body size perception in men. As noted earlier, perception involves the processing of information in the brain and, therefore, numerous subjective factors could affect how an individual judges the size of an object, especially his or her body. ${ }^{4}$ Some of these subjective factors likely include the affective and attitudinal dimensions of body size perception, which have been traditionally studied as body dissatisfaction. ${ }^{1}$ Size distortion and body dissatisfaction have typically been conceived as separate aspects of body image disturbance, and studies of the relationship between the two have revealed disparate findings. ${ }^{8}$ More recently, some investigators have begun using advanced psychophysical techniques that allow for the separate measurement of the perceptual factors of body size estimation from the subjective/affective factors. ${ }^{4}$ This is important because contemporary research indicates that these two classes of factors are largely independent of one another in most nonclinical populations. ${ }^{4}$

Another difficulty that contributes to the disparate findings in body image research on men is that, without exception, the evidence is based on cross-sectional research that compares men in different age groups. ${ }^{9}$ Perhaps not surprisingly, longitudinal research that compares the same individuals as they age is missing. This is problematic, as men at different ages are from different generations, with particular life experiences that differ across generations. Much of the research on men's body size perception has been conducted on young, college-aged individuals, where the age range is relatively restricted. ${ }^{9}$

An additional difficulty in the interpretation of findings is the disparate methodologies that have been used to measure estimations of body size. Many of these methodologies, especially those used in earlier research, have either undocumented or poor psychometric properties. ${ }^{10,11}$ These included assessing distortion by showing subjects photographs or figural drawings illustrating the frontal perspective of a body, and having subjects select the figure or photograph closest to their perceived size. In other studies, subjects adjusted lights or bars spaced horizontally to judge their perceived size. ${ }^{10}$ Later studies used the video-distortion technique in which a digital image of a subject can be manipulated to become wider or thinner. ${ }^{10}$

Lastly, most research on how weight status affects perception of men has focused on how overweight and obese participants compare to normal participants. ${ }^{7}$ In these studies, participants are typically dichotomized into normal-weight and overweight/obese groups, and their differences are examined. In some of the early studies, normative data developed by the Metropolitan Life Insurance Company ${ }^{12}$ were used to categorize subjects into weight categories. A subject was judged as obese if they weighed $20 \%$ over the norm. In later studies, the body mass index $\left(\mathrm{BMI}=\mathrm{kg} / \mathrm{m}^{2}\right)$ was used to classify subjects' weight categories. ${ }^{4}$ Placing subjects into discrete weight categories revealed limited information about how perception is affected within a range of weights. As a personal mea culpa, this author published several of the early studies on body image perception in obese individuals, wherein the analyses have included this limitation. ${ }^{13-18}$ Later, I will include some additional post hoc analyses on data from recent studies that address this limitation and provide 
additional information about body size estimation accuracy more precisely within weight ranges.

The early research on the role of obesity and body size perception in men was marked by inconsistent findings. A review of the research findings in this area conducted between 1967 and 1986 found contradictory reports, with about equal numbers of studies finding that obese subjects overestimated their body size, and with the remaining studies finding that obese subjects were relatively accurate in size estimations. ${ }^{11}$ By contrast, most of these early studies found that normal weight subjects were accurate in estimating their body size. All of these early studies looked at the two weight groups dichotomously and therefore, as noted earlier, the precise relationship between body size and size perception was unknown. These early studies were conducted with a variety of body size estimation techniques, many of which have subsequently proven to involve methodologies that lack good psychometric properties, including documented reliability or validity. ${ }^{11}$

Beginning in 1987, advances in technology permitted more sophisticated ways of measuring body size estimation. Some researchers began using the aforementioned video-distortion techniques in their research. ${ }^{4}$ Most frequently, participants viewed an image of themselves on a television or a video monitor, in which their image was initially too thin or too wide. Participants were able to adjust the width of the image to match the size they perceived themselves to be. This is what classical psychophysics defines as the method of adjustment (MOA). ${ }^{4} \mathrm{~A}$ body perception index (BPI) can be calculated by examining the discrepancy between the subjects' estimated size and their actual size:

$$
\mathrm{BPI}=(\text { estimated size/actual size }) \times 100 .
$$

For example, if an individual adjusts the width of his or her image (perceived size) to $110 \%$ of his or her actual size, the BPI would be calculated as:

$$
(110-100) \times 100, \mathrm{BPI}=+10,
$$

or an overestimation of $10 \%$. Similarly, an adjustment of an image to $85 \%$ of the subject's actual size would represent an underestimation of $15 \%$ :

$$
(85-100) \times 100
$$

or $\mathrm{BPI}=-15$.

Thompson et $\mathrm{al}^{19}$ described how research performed in our laboratory in the last 18 years has:

[...] reenergized the study of perceptual processes of body image disturbance. The basic goal $[\ldots]$ is to separate sen- sory from nonsensory factors. Sensory, in the case of size perception, refers to the responses of the visual system (retinal, visual cortex); nonsensory refers to other inputs in the mind that help to interpret the visual input. ${ }^{19}$

Some of the earliest of these studies also employed a signal detection theory (SDT) analysis, which uses psychophysical methods to allow for the separation of sensory and nonsensory factors. ${ }^{19}$ Thompson et al also describe how:

Through a mathematical analysis of the correct responses and errors in a visual detection task, two measures emerge: sensory sensitivity, which is the ability to detect the sensory stimulus, and response bias, which is the tendency to interpret the stimulus in a distorted way. ${ }^{19}$

Briefly, in SDT, participants are presented with a discrete image of their bodies, which is either an accurate size or is distorted - either too wide or too thin. ${ }^{19}$ Participants are required to judge whether the image was accurate or distorted with respect to size on each of several trials. Separate measures are obtained, reflecting how sensitive the participants are in detecting size distortion in their body image (sensory aspect), and a measure of response bias is also obtained (nonsensory factors). This latter measure indicates whether participants have a response bias or a tendency to report that an accurately portrayed image of their body is distorted, either being too wide or too thin. ${ }^{4}$ The use of these more advanced psychophysical techniques dramatically changed how body image perception was studied because it allowed for the separation of the sensory and nonsensory components, which are theoretically independent of one another. Gardner and Brown ${ }^{4}$ give a more detailed explanation of how SDT is applicable to a body size estimation task.

Several studies on body size perception were conducted in our laboratory during the late 1980s using the videodistortion technique in conjunction with MOA and SDT methodologies. ${ }^{13-18}$ Participants included both men and women, who were university students and/or participants from the local community. Regrettably, no specific age data were reported in these studies, but the recollection of this author is that the majority of subjects ranged from 18-49 years old. The findings describe the accuracy of body size estimations in obese individuals as compared to normal weight controls. As noted earlier, dichotomization into two weight categories does not permit the accurate determination of the precise relationship between a range of BMI values and body size perception. However, it does address the question of how accurately men who are obese (as opposed to being of 
normal weight) perceive their body size. In only one instance was a sex difference found, suggesting that when it comes to perceiving one's body size with these methodologies, both obese and normal-weight males and females typically performed the same. ${ }^{13-17}$

The first study to measure the perception of body image in obese versus normal-weight participants using both the MOA and an SDT analysis was conducted by Gardner et al. ${ }^{13}$ The video-distortion methodology was used with subjects viewing their video image on a computer monitor. The participants included 38 males and females from a local university and from the surrounding community, half of whom were categorized as obese. Subjects viewed their images in both regular street clothes and with minimal clothing. Men's data were not analyzed separately, as no significant sex differences were found. Data from the MOA task, which calculated subjects' BPI, revealed that obese subjects were more likely to overestimate their body size when compared to the normal weight controls. The SDT analysis found no overall difference between obese and normal-weight subjects in their ability to detect changes in their body size. However, obese subjects were better at detecting distortion in their body size in an image that was thinner than their actual size, but they were poorer in detecting distortion in images that were wider than their actual size. There was no response bias difference between the two weight groups. In addition, the two clothing conditions did not affect either the sensory or the nonsensory aspects of the body image task.

In a follow-up study by Gardner et $\mathrm{al}^{14}$ eleven obese and eleven normal-weight males were again compared using the video-distortion methodology with the SDT and MOA tasks. Obese subjects overestimated body width more often than did the normal-weight subjects, although the difference did not reach significance. Consistent with the earlier study, an SDT analysis revealed that there was no difference between weight groups in terms of their sensitivity to detect distortions in their body size. ${ }^{14}$

A subsequent study compared ten obese and eleven normal-weight males on body size estimation, again including the video-distortion methodology and MOA and SDT tasks. ${ }^{15}$ The subjects were recruited from the local university community. On the MOA task, obese subjects overestimated not only the size of their whole body, but individual body regions including the face, waist, and thighs. In the SDT task, obese subjects were poorer in detecting size distortion in their bodies, including their whole body, as well as with respect to the individual body regions. As occurred in one previous study, ${ }^{14}$ the detection of distortion by obese males in images that were too wide was more difficult than for images that were too thin, when compared to controls of normal weight. These findings may hold clinical significance. A decreased sensory ability to detect size distortion when the images are too large may result in obese men being less likely to engage in behaviors that lead to weight loss. Further studies are needed to explore these sensory sensitivity differences between obese and normal-weight men.

Another study conducted in our laboratory compared 21 obese and 23 normal-weight subjects from the local community, including both sexes, on a body size estimation task using the video-distortion technique and the MOA. ${ }^{16}$ The subjects made judgments of their body size in the presence and absence of a full-length mirror. Although obese subjects overestimated their body size by about $15 \%$, as compared to the control group who overestimated by about $11 \%$, the difference did not reach significance. Both weight groups were more accurate in estimating their body size when viewing their image in a mirror. As in previous studies, no sex differences were found.

In a further study conducted in our laboratory, we again used the video-distortion methodology to compare body size estimations in 20 obese and 20 normal-weight subjects, including equal numbers of men and women. ${ }^{17}$ In this study, the psychophysical method of constant stimuli was employed. In this methodology, subjects viewed their bodies at eleven discrete levels of distortion ranging between $\pm 20 \%$ too wide or too thin. This methodology allows for the computation of the point of subjective equality (PSE), which represents the amount of body size distortion, in percentage values, of over- or underestimation. ${ }^{17}$ The PSE is the size of a subject's body image that is judged subjectively as representing their actual body size. The PSE thus represents the amount of distortion present in subjects' judgment of their body size. Being a measure of subjective size, it is reflective of nonsensory affective and attitudinal factors. In addition, the difference threshold (henceforth referred to as the just noticeable difference [JND]) is also measured. This is the amount of change in body size distortion necessary for the subject to detect a change in body size $50 \%$ of the time. This measures how sensitive the subject is to detecting changes in his or her body size, and represents the sensory component of body size estimation. In psychophysics, these two factors are largely independent of one another. That is, a subject's ability to detect changes in his or her body size is not necessarily related to feelings of body dissatisfaction. ${ }^{14}$ On the MOA task, both obese and control subjects were accurate in estimating their body size. ${ }^{17}$ With the constant 
stimuli task, subjects' PSE values indicated that they were very accurate in judging their body size. No differences were found between obese and control subjects, or between sexes. ${ }^{17}$ As expected, no differences were found between weight groups or sexes on the JND, indicating equivalent sensory sensitivity for detecting changes in body size for all groups. This was the first study to quantify the JND in a body image perception task. An average distortion of 7.3\% was necessary for subjects to detect a distortion (either wider or thinner) in their body size $50 \%$ of the time.

In a subsequent study, we again compared body size estimations in both obese and normal-weight male subjects. ${ }^{18}$ Obese subjects overestimated their body size slightly more than the normal-weight subjects, although the difference did not reach significance. This is one of the rare instances in our laboratory where a significant sex difference in body size estimation accuracy was found, with males overestimating their body size more than females. Subjects estimated their body size before and after a meal. Interestingly, obese subjects estimated their body size to be wider after eating a large meal, while normal-weight subjects estimated their body size to be thinner. It may be that obese subjects are more acutely aware of sensory cues associated with satiety, such as stomach extension or how tightly clothes feel against the body.

Smeets ${ }^{21}$ has argued against a body image methodology in which a subject is presented with a stimulus of his or her body, and is then asked to make a judgment about his or her body size. Because subjects do not directly perceive their bodies at the time of assessment, the author feels that memory, rather than perception of body size, is measured. Farrell et $\mathrm{al}^{22}$ have developed a mirror-based assessment method that they believe measures perception rather than one's memory of body size. Subjects viewed a projected image of themselves that is the same size as a subject's reflection in a mirror. Subjects look in the mirror as they adjust the size of the projected image. When used with women, size estimations are significantly larger than are usually obtained with the methods described earlier. No known studies with men have used this assessment method, and whether comparable results would be found with men remains unknown. Whether body perception and body memory are separable components of body image also remains in question. It should be noted that in psychophysics, no such distinction is made when making judgments of stimuli. ${ }^{23}$ For example, in psychophysics, subjects are sometimes asked to judge whether two objects that are identical in size differ in weight. In this case, it is a "memory" of the two weights that is being judged, and sensory and nonsensory components can be separately measured. Body image reflects, in part, our internal view of how we think we look, and memory is an important aspect of that conception.

One popular method for assessing body dissatisfaction is through the use of figural drawing scales. ${ }^{24}$ These scales consist of a series of drawings of frontal outline images of the human form, and they range from thin to obese. Subjects are typically asked to select the image that most accurately represents their current perceived size, and the size they would ideally like to be. The discrepancy between these two is a measure of body dissatisfaction. These scales are popular with researchers because they are easy to use, and the data can be collected in a group setting. Numerous scales have been developed, and those with documented psychometric properties have been reviewed by Gardner and Brown. ${ }^{24}$

While nearly all of these scales are used to measure body dissatisfaction exclusively, a scale recently developed in our laboratory called the Body Image Assessment Scale (BIAS-BD) can be used to measure both body dissatisfaction and the accuracy of body size estimations. ${ }^{25}$ Seventeen figural drawings were created. The drawings represent body sizes ranging from thin to obese, ranging from $40 \%$ below the United States adult average to $40 \%$ above average. Drawings are based on the known anthropometric body measurements of body regions, including the shoulder, chest, waist, hip breadth, thigh breadth, and upper leg breadth. The scale demonstrated good psychometric properties, including testretest reliability and convergent validity. ${ }^{25}$

Using this BIAS-BD scale, we collected data on weight status and body size estimation error on a sample of 66 college-age men. ${ }^{25}$ Age data were not collected on the sample in order to ensure anonymity. Men had an average weight of $75.0 \mathrm{~kg}$ (standard deviation $[\mathrm{SD}]=14.9 \mathrm{~kg}$; range $=54-76 \mathrm{~kg}$ ) and a BMI of 23.5 ( $\mathrm{SD}=4.53$; range $=16.3-38.7)$. Men were relatively accurate in estimating their body size, overestimating by $+3.29 \%$ ( $\mathrm{SD}=18.66 \%$ ), an overestimation that was not significantly greater than zero. The correlation between BMI and accuracy of body size estimations was $r=-0.02$, a value not significantly different from zero. This lack of correlation shows that men's accuracy in estimating their body size is independent of their actual body size, as reflected in a range of BMI values.

Another study used what is called a somatomorphic matrix to measure body image perception in young men in their early to mid 20s. ${ }^{26}$ Subjects included 24 Austrian, 54 French, and 65 US men. The matrix consists of a computerized library of 100 images of men representing ten levels of fatness and 
ten levels of muscularity. The outline images were created by a graphic artist based on actual photographs of men. Men were relatively accurate in perceiving the amount of body fat in their body, with only modest differences noted between countries. Men in all three countries perceived themselves to be significantly more muscular than they actually were, although in terms of absolute values, the muscularity distortion was modest.

More recently, in our laboratory, we introduced a more advanced psychophysical technique called adaptive probit estimation (APE) to measure body size estimation accuracy. ${ }^{27}$ APE uses a methodology similar to that of the method of constant stimuli described earlier. Computer software was developed that presents numerous static video images of a subject's body, and these images are distorted to be either too wide or too thin. ${ }^{27}$ Subjects must judge whether each video image is too wide or too thin. The amount of distortion is adjusted over eight blocks of 40 trials, according to how the subject performs on the body size estimation task. A psychometric function is estimated, and measures of body image perception are determined. As in the method of constant stimuli, separate measures of the PSE and JND are found. A more detailed description of the APE methodology is beyond the scope of this paper and can be found in Gardner and Boice ${ }^{28}$ and Gardner and Brown. ${ }^{4}$

Although APE has not been used in any studies comparing obese and normal-weight subjects it has, in conjunction with the MOA, been used in several studies in our laboratory in which college-age men were subjects. ${ }^{17,28}$ By retroactively examining these data, it has been possible to determine the correlation between young men's actual body size and the accuracy of their body size estimations. For the APE methodology, the PSE is used as a measure of body size estimation accuracy, while with the MOA, the BPI is used. Both the PSE and the BPI reflect the percentage by which male subjects over- or underestimate their body size.

In a recent study, we measured the accuracy of body size estimations using both the BIAS-BD figural drawing scale, as well as the video-distortion methodology and APE methodology. ${ }^{28}$ Participants included 26 college-age men with an average age of 23.35 years $(\mathrm{SD}=7.63$ years; range $=18-57$ years). Subjects had an average weight of $80.4 \mathrm{~kg}(\mathrm{SD}=17.3 \mathrm{~kg}$; range $=54-129 \mathrm{~kg})$, and an average BMI of 25.96 ( $\mathrm{SD}=5.01$; range $=17.2-39.3)$. Overall, men were accurate in estimating their body size both with the figural drawing scale and with the video distortion software. For the BIAS-BD scale, men overestimated their body size by $M=+4.04 \%(\mathrm{SD}=5.17 \%)$. For the video distortion software, two estimates of body size distortion were obtained; the PSE showed that men slightly overestimated their body size by $+0.32 \%(\mathrm{SD}=5.17 \%)$, and with the MOA, men underestimated their body size by $M=-1.98 \%$ (SD =6.25\%). An average of these three measures showed that, overall, men were accurate in estimating their body size, with an average overestimation of $<1.0 \%(M=0.80 \%$; SD $=7.56 \%)$, a difference that was not significantly different from zero. A retroactive examination was made of the correlation between BMI and this average level of body size distortion. The obtained correlation was $r=-0.01$, again indicating no relationship between men's body size and how accurately they estimated their body size.

As noted earlier, the APE methodology also allows for the measurement of the JND, which reflects the amount of change in body size necessary for a subject to detect that change $50 \%$ of the time. ${ }^{4}$ The JND reflects the sensory sensitivity of the subjects when detecting changes in their body size. ${ }^{4}$ For the men in this study, the average (root mean square) JND was 2.64. This indicates that on average, men required a $2.64 \%$ change in their body size to detect that change onehalf of the time. The correlation between body size (BMI) and JND values was $r=-0.11$, a correlation not significantly different from zero. This lack of relationship showed, for the first known time, that men's sensory ability to detect changes in their body size is not related to their body size.

The reanalysis of data from these two studies are the only known instances in which the accuracy in estimations of body size has been correlated with actual body size (BMI in these analyses). Both studies provide support for the notion that how accurately men estimate their body size is not significantly related to their actual body size.

\section{Sociocultural differences}

A recent trend has emerged, where the theoretical framework used to view body image is known as the sociocultural perspective. ${ }^{29}$ From this perspective, sociocultural ideals and pressures are of the upmost importance in the origins of body image. Although it does not deny the importance of individual characteristics, the focus is primarily on the influences that arise within a specific sociocultural environment. This includes aspects of the physical environment, as well as the social and cultural practices, norms, and beliefs of a society. ${ }^{29}$ These influences are often different for men and women. ${ }^{29}$

As noted previously, the important role of body image in the development and maintenance of eating disorders has led to the vast majority of studies examining sociocultural influences on body image among women. ${ }^{30}$ Sociocultural 
influences also affect young men. In those cultures where Western influences are more prominent, there is an increasing desire for young men to have a mesomorphic and muscular V-shaped body. ${ }^{30}$ This includes broad shoulders, a flat but muscular stomach, and a narrow waist and hips. ${ }^{29}$ Since this ideal is nearly impossible to attain, the result is increasing levels of body dissatisfaction in men, similar to what is occurring in women. ${ }^{30} \mathrm{~A}$ separate issue - the one that is focused on in this article - is whether there are differences among different cultures in how accurately men perceive their body size. An exhaustive review of the literature in this area is beyond the scope of this paper. Instead, a sample of contemporary studies examining body perception in men from a variety of different cultures will be presented.

In recent years, the focus of research on body image perception in men has increasingly included studies in which men are asked whether they consider themselves to be in one of a discrete number of specific categories of weight. This usually involves four to five categories ranging from underweight to obese. Subjects' BMI is then calculated, and calculations are made regarding how accurately subjects placed themselves into the appropriate weight category. Perhaps not too surprisingly, men are often inaccurate in rating themselves, often more so than women. ${ }^{31-37}$ Findings have shown that men who are underweight or are of normal weight are usually accurate in classifying their weight status, while overweight and obese men underestimate their body size by choosing a weight category thinner than their actual size. Studies from several countries have documented this finding. Underestimations of body size in obese and overweight subjects have been reported in studies with men from the US, ${ }^{31}$ Pakistan, ${ }^{32}$ Australia, ${ }^{33}$ Spain, ${ }^{34}$ Peru, ${ }^{35}$ Brazil, ${ }^{36}$ and South Africa. ${ }^{37}$

One study included 8,115 young men enrolled in undergraduate classes who were aged $17-30$ years. ${ }^{38}$ Subjects were from 22 countries constituting five geopolitical/ economic areas including north-western Europe and the US, central and eastern Europe, the Mediterranean, Pacific Asia, and South America. Rather than use BMI values to identify overweight and obesity, men's BMI values were categorized into deciles relative to each country sample. Interestingly, very few men who are below the seventh decile (70th percentile) based on their country norms feel overweight, and even many of those in the ninth or tenth decile do not describe themselves as overweight. Inaccurate perceptions of overweight were very consistent across the 22 countries within the five geopolitical/economic areas.

The tendency for overweight and obese men to rate themselves as thinner than they actually are may be an instance whereby men exhibit a greater denial of their true body size, perhaps because of demand characteristics or other social pressures. It should be recalled that sex differences were rarely found in our laboratory when using psychophysical techniques to measure body size estimations. ${ }^{13-18}$

Socioeconomic status (SES) may also play an important role in perceptions of overweight. Studies have reported fewer accurate perceptions of overweight in low SES groups, including those in the US, ${ }^{39}$ the $\mathrm{UK},{ }^{40}$ and Peru. ${ }^{41}$ Individuals from low SES groups frequently eat foods that are less expensive, but are also less healthy and more likely to cause weight gain. ${ }^{39-41}$

It is important to note that this methodology asks men to "report" which weight category they believe they fall into, and this may reflect a response bias as much, or perhaps more than, a true reflection of their abilities to accurately perceive their body size. ${ }^{10}$ Demand characteristics or other sociocultural factors could result in men having a tendency to give a rating of themselves that underestimates their actual body size, and these could be tangential to their actual capabilities to accurately perceive their body size.

Other contemporary studies have used recently developed figural drawing scales to measure how accurately men perceive their body size in different cultures. ${ }^{42-44}$ Subjects are shown a series of outlines or photos of the human body ranging from thin to obese, and they are asked to select the one that most closely represents their actual size. One study examined the accuracy of perceived body size in a representative sample of 3,215 overweight and obese males over the age of 15 years who were residents in the 15 European Union countries. ${ }^{42,43}$ Subjects selected their perceived body size from a figural drawing scale with nine depictions of the body, representing BMI values ranging from very thin to obese. Overall, two-thirds of the overweight and obese males underestimated their body size, with younger males underestimating their size more frequently than middle-aged or older men. Obese men were particularly inaccurate, with three-quarters underestimating body size. Increasing age was inversely related to underestimation, with men older than 55 years being the most accurate in estimating their body size. The factors that correlated positively with men's underestimation included obesity, having a postsecondary education, being a current smoker, engaging in frequent exercise, and living in a Mediterranean country. Overall, men were less accurate than women in accurately perceiving their body size. ${ }^{43}$

Another recent study used nine figural drawings ranging from thin to obese to examine body size estimation accuracy in 55 male Nigerian undergraduates ranging in age from 
21-29 years $(M=22.9$ years; $\mathrm{SD}=1.88$ years $) .{ }^{44} \mathrm{BMI}$ values averaged $23.45(\mathrm{SD}=2.69)$. Men with a $\mathrm{BMI}$ in the normal weight range correctly perceived their body size, while those in the overweight category underestimated their weight and perceived themselves to be in the normal-weight category.

One study used photographs of men ranging from very thin to obese, rather than figural drawings. ${ }^{45}$ Subjects were 65 men ranging in age from 19-77 years, with an average age of 36.8 years ( $\mathrm{SD}=10.9$ years). Men selected the picture they felt corresponded most closely to their own body size. As previously found, men who were thin or of normal weight accurately identified a picture close to their normal weight, while overweight and obese men underestimated their weight. This latter group of men also misidentified the pictures of other overweight men as being of normal weight.

There is an increasing awareness that perception of body shapes and sizes is influenced by cultural factors..$^{46,47}$ The word "overweight" denotes an excess in body weight relative to a standard that is often influenced by cultures. ${ }^{45}$ Even within a given culture, such judgments can vary. In the US, what might otherwise be viewed as obesity in most men would be judged as a normal body size for a professional football lineman.

The influence of cultural values on weight perception was demonstrated recently in a study in Nairobi, Kenya in which researchers used an 18-drawing figural scale with 2,669 men with a mean age of 42 years who had a low income and resided in slums. ${ }^{48}$ The proportion of men who perceived themselves to be overweight or obese was greater than the actual number falling in that category. Of the subjects classified as overweight or obese, about one-half underestimated their BMI. Overall, there was a strong preference for a larger body size. The authors noted that in some societies in sub-Saharan Africa, a larger body size is seen to reflect good health and higher social status and, therefore, may be considered desirable. ${ }^{48}$

Similar findings were found in a recent study of a representative sample of 6,941 men in South Korea, of whom 3,068 were between the ages of 20 years and 39 years. ${ }^{49}$ Age was not associated with accuracy of weight perception in men. While about two-thirds accurately judged their weight category, nearly $25 \%$ judged their weight category as smaller than they actually were. Men of a low SES had a higher prevalence of underestimating their weight. As in sub-Saharan Africa, a larger body size in Korea has traditionally been desired as a symbol of health, wealth, and social stature. While this preference has been weakening somewhat in recent years, many Korean men still view larger body sizes a symbols of masculinity and power. ${ }^{49}$

\section{Conclusion}

Early studies comparing body size estimations of obese and normal-weight subjects revealed inconclusive findings. The later introduction of improved computerized video-distortion technologies and psychophysical methodologies for assessing body size perception has helped clarify the respective roles of sensory and nonsensory/affective variables in body size perception. This is important because these two classes of variables are largely independent of one another. ${ }^{20}$

The majority of contemporary studies using these psychophysical techniques found no significant difference between obese and normal-weight men in estimating their body size, although there was a tendency for obese men to overestimate their size. However, two studies in our laboratory ${ }^{17,18}$ found that obese men are significantly poorer at detecting images of themselves that were too wide, as compared to too thin - a finding that may have clinical significance. Importantly, when including men within a range of weight values, the correlation between errors in body size estimation and body size is close to zero. Further investigations using methodological tools that allow the separate investigation of sensory and nonsensory factors in body image perception are very much needed.

More recent studies have frequently asked men to categorize their weight into one of several discrete categories ranging from thin to overweight. Most men who are thin or who are in the normal weight range are accurate in categorizing themselves, while overweight and obese men consistently report underestimations of their size. ${ }^{31-37}$ These findings have been consistent across several nationalities. Distinct differences have also been found between cultures where smaller versus larger body sizes are seen as preferred. ${ }^{48,49}$

Methodological limitations in studies on body image have often restricted the conclusions that can be drawn. ${ }^{10}$ In addition, nearly all contemporary studies use BMI as a measure of body size. ${ }^{10}$ Using BMI alone to define obesity has numerous limitations, with some men with large muscle mass, such as athletes, being incorrectly classified as overweight or obese..$^{50}$ Moreover, many studies rely on selfreports of height and weight. Previous studies have shown that adults who self-report tend to underreport their body weight, and they overreport their body height, with resulting inaccuracies in calculated BMI values. ${ }^{50}$ Alternative indices of weight status include percentile charts that use weight and height as a reference, direct measurement of subcutaneous 
fat, assessment of total body fat via skin-fold measurements, body density, and lean body mass measurements. ${ }^{51}$ There is also a long held need for longitudinal studies that detail how body image perception changes during the aging process.

\section{Acknowledgments}

The series of research studies by Gardner et al that compare body image in obese versus normal-weight subjects was supported by grant 08197-06 funded by the Division of Research Resources and the National Institute of Mental Health to Rick M Gardner. The body image software described in the studies by Gardner et al is available at no cost from the author.

\section{Disclosure}

The author reports no conflicts of interest in this work.

\section{References}

1. Thompson JK. Introduction: Body image, eating disorders, and obesity - an emerging synthesis. In: Thompson JK, editor. Body Image, Eating Disorders, and Obesity. Washington, DC: American Psychological Association; 1996:1-22.

2. Sciacca JP, Melby CL, Hyner GC, Brown AC, Femea PL. Body mass index and perceived weight status in young adults. J Community Health. 1991;16(3):159-168.

3. Lemon SC, Rosal MC, Zapka J, Borg A, Andersen V. Contributions of weight perceptions to weight loss attempts: differences by body mass index and gender. Body Image. 2009;6(2):90-96.

4. Gardner RM, Brown DL. Measurement of the perceptual aspects of body image. In: Greene SB, editor. Body Image: Perceptions, Interpretations and Attitudes. New York, NY: Nova Science Publishers; 2011:81-102.

5. Thompson JK, Heinberg LJ, Altabe MN, Tantleff-Dunn S. Exacting Beauty: Theory, Assessment, and Treatment of Body Image Disturbance. Washington, DC: American Psychological Association; 2004:290-293.

6. Garner DM. Body image and anorexia nervosa. In: Cash TF, Pruzinsky T, editors. Body Image: A Handbook of Theory, Research, and Clinical Practice. New York, NY: Guilford Press; 2002:295-303.

7. Jones W, Morgan J. Eating disorders in men: a review of the literature. J Public Ment Health. 2010;9(2):23-31.

8. Gardner RM, Tockerman YR. Body dissatisfaction as a predictor of body size distortion: a multidimensional analysis of body image. Genetic, Social and General Psychology Monographs. 1993;119(1): $125-145$

9. Gogan S. Body image development in adulthood. In: Cash TF, Smolak L, editors. Body Image: A Handbook of Science, Practice, and Prevention. 2nd ed. New York, NY: The Guilford Press; 2011:93-100.

10. Gardner RM. Methodological issues in assessment of the perceptual component of body image disturbance. Br J Psychol. 1996;87(Pt 2): 327-337.

11. Wright EJ, Whitehead TL. Perceptions of body size and obesity: a selected review of the literature. J Community Health. 1987;12(2-3): $117-129$

12. About the "Metropolitan Life" tables of height and weight [webpage on the Internet]. Hall SB; 2008 [updated May 26, 2008]. Available from: http://www.halls.md/ideal-weight/met.htm. Accessed July 17, 2014.

13. Gardner RM, Martinez R, Sandoval Y. Obesity and body image: an evaluation of sensory and non-sensory components. Psychol Med. 1987;17(4):927-932.
14. Gardner RM, Martinez R, Espinoza T. Psychophysical measurement of body image of self and others in obese subjects. $J$ Soc Behav Pers. 1987;2(2 Pt 1):205-217.

15. Gardner RM, Martinez R, Espinoza T, Gallegos V. Distortion of body image in the obese: a sensory phenomenon. Psychol Med. 1988;18(3):633-641.

16. Gardner RM, Gallegos V, Martinez R, Espinoza T. Mirror feedback and judgments of body size. J Psychosom Res. 1989;33(5):603-607.

17. Gardner RM, Morrell JA, Watson DN, Sandoval SL. Subjective equality and just noticeable differences in body-size judgments by obese persons. Percept Mot Skills. 1989;69(2):595-604.

18. Gardner RM, Espinoza T, Urrutia R, Morrell J, Gallegos V. The effect of hunger on body image in the obese: feeling full, feeling fat. Psychiatr Forum. 1990;15:57-62.

19. Thompson JK, Heinberg LJ, Altabe MN, Tantleff-Dunn S. Exacting Beauty: Theory, Assessment, and Treatment of Body Image Disturbance. Washington, DC: American Psychological Association; 2004:291.

20. Taner WP, Swets JA. A decision-making theory of visual detection. Psychol Rev. 1954;61(6):401-409.

21. Smeets MA. The rise and fall of body size estimation research in anorexia nervosa: a review and reconceptualization. European Eating Disorders Review. 1997;5(2):75-95.

22. Farrell C, Shafran R, Fairburn CG. Body size estimation: testing a new mirror-based assessment method. Int J Eat Disord. 2003;34(1):162-171.

23. Kingdom FAA, Prins N. Psychophysics: A Practical Introduction. New York: Elsevier; 2010

24. Gardner RM, Brown DL. Body image assessment: a review of figural drawing scales. Pers Individ Dif. 2010;48(2):107-111.

25. Gardner RM, Jappe LM, Gardner L. Development and validation of a new figural drawing scale for body-image assessment: the BIAS-BD. J Clin Psychol. 2009;65(1):113-122.

26. Pope HG, Gruber AJ, Mangweth B, et al. Body image perception among men in three countries. Am J Psychiatry. 2000;157(8): 1297-1301.

27. Gardner RM, Boice R. A computer program for measuring body size distortion and body dissatisfaction. Behav Res Methods Instrum Comput. 2004;36(1):89-95.

28. Gardner RM, Brown DL. Comparison of video distortion and figural drawing scale for measuring and predicting body image dissatisfaction and distortion. Pers Individ Dif. 2010;49(7):794-798.

29. Tiggemann M. Sociocultural perspectives on body image. In: Cash TF, editor. Encyclopedia of Body Image and Human Appearance. Burlington, MA: Elsevier Science; 2012:758-765.

30. Thompson JK, Heinber LJ, Altabe M, Tantleff-Dunn S. Sociocultural theory: the media and society. In: Thompson JK, Heinberg LJ, Altabe MN, Tantleff-Dunn S, editors. Exacting Beauty: Theory, Assessment, and Treatment of Body Image Disturbance. Washington, DC: American Psychological Association; 1999:85-124.

31. Hemiup JT, Carter CA, Fox CH, Mahoney MC. Correlates of obesity among patients attending an urban family medical center. $J$ Natl Med Assoc. 2005;97(12):1642-1648.

32. Bhanji S, Khuwaja AK, Siddiqui F, Azam I, Kazmi K. Underestimation of weight and its associated factors among overweight and obese adults in Pakistan: a cross sectional study. BMC Public Health. 2011;11:363.

33. Donath SM. Who's overweight? Comparison of the medical definition and community views. Med J Aust. 2000;172(8):375-377.

34. Gutiérrez-Fisac JL, López García E, Rodriguez-Artalejo F, Banegas Banegas JR, Guallar-Castillón P. Self-perception of being overweight in Spanish adults. Eur J Clin Nutr. 2002;56(9):866-872.

35. Jacoby E, Goldstein J, López A, Núñez E, López T. Social class, family, and life-style factors associated with overweight and obesity among adults in Peruvian cities. Prev Med. 2003;37(5):396-405.

36. Araújo CL, Dumith SC, Menezes AM, Hallal PC. [Measured weight, self-perceived weight, and associated factors in adolescents]. Rev Panam Salud Publica. 2010;27(5):360-367. Portuguese. 
37. Peltzer K, Pengpid S. Body weight and body image among a sample of female and male South African university students. Gender and Behaviour. 2010;10(1):4509-4522.

38. Wardle J, Haase AM, Steptoe A. Body image and weight control in young adults: international comparisons in university students from 22 countries. Int J Obes (Lond). 2006;30(4):644-651.

39. Chang VW, Christakis NA. Self-perception of weight appropriateness in the United States. Am J Prev Med. 2003;24(4):332-339.

40. Wardle J, Griffith J. Socioeconomic status and weight control practices in British adults. J Epidemiol Community Health. 2001;55(3):185-190.

41. Loret de Mola C, Pillay TD, Diez-Canseco F, Gilman RH, Smeeth L, Miranda JJ. Body mass index and self-perception of overweight and obesity in rural, urban and rural-to-urban migrants: PERU MIGRANT study. PLoS One. 2012;7(11):e50252.

42. Madrigal H, Sánchez-Villegas A, Martínez-González MA, et al. Underestimation of body mass index through perceived body image as compared to self-reported body mass index in the European Union. Public Health. 2000;114(6):468-473.

43. Sánchez-Villegas A, Madrigal H, Martínez-González MA, et al. Perception of body image as indicator of weight status in the European union. J Hum Nutr Diet. 2001;14(2):93-102.

44. Maruf FA, Akinpelu AO, Nwankwo MJ. Perceived body image and weight: discrepancies and gender differences among University undergraduates. Afr Health Sci. 2012;12(4):464-472.
45. Harris CV, Bradlyn AS, Coffman J, Gunel E, Cottrell L. BMI-based body size guides for women and men: development and validation of a novel pictorial method to assess weight-related concepts. Int J Obes (Lond). 2008;32(2):336-342.

46. Rudofsky B. The Unfashionable Human Body. New York, NY: Doubleday; 1972.

47. Allon N. The stigma of overweight in everyday life: obesity in perspective. In: Bray GA, editor. Fogarty International Center Series on Preventive Medicine. Washington, DC: US Government Printing Office; 1973:83-102.

48. Ettarh R, Van de Vijver S, Oti S, Kyobutungi C. Overweight, obesity, and perception of body image among slum residents in Nairobi, Kenya, 2008-2009.

49. Joh HK, Oh J, Lee HJ, Kawachi I. Gender and socioeconomic status in relation to weight perception and weight control behavior in Korean adults. Obes Facts. 2013;6(1):17-27.

50. Dekkers JC, van Wier MF, Hendriksen IJ, Twisk JW, van Mechelen W. Accuracy of self-reported body weight, height and waist circumference in a Dutch overweight working population. BMC Med Res Methodol. 2008;8:69.

51. Ross WD, Crawford SM, Kerr DA, Ward R, Bailey DA, Mirwald RM. Relationship of the body mass index with skinfolds, girths, and bone breadths in Canadian men and women aged 20-70 years. Am J Phys Anthropol. 1988;77(2):169-173.
Psychology Research and Behavior Management

\section{Publish your work in this journal}

Psychology Research and Behavior Management is an international, peerreviewed, open access journal focusing on the science of psychology and its application in behavior management to develop improved outcomes in the clinical, educational, sports and business arenas. Specific topics covered include: Neuroscience, memory \& decision making; Behavior

\section{Dovepress}

modification \& management; Clinical applications; Business \& sports performance management; Social and developmental studies; Animal studies. The manuscript management system is completely online and includes a quick and fair peer-review system. Visit http://www.dovepress. com/testimonials.php to read real quotes from published authors. 